\title{
Meeting report American Society for Clinical Oncology 40th Annual Meeting,
New Orleans, LA, 5-8 June 2004: breast cancer highlights
} Mark L Sundermeyer and Lori J Goldstein

Fox Chase Cancer Center, Philadelphia, Pennsylvania, USA

Corresponding author: Lori J Goldstein, LJ_Goldstein@fccc.edu

Published: 6 October 2004

Breast Cancer Res 2004, 6:275-278 (DOI 10.1186/bcr944)

(c) 2004 BioMed Central Ltd

The American Society for Clinical Oncology (ASCO) held its 40th annual meeting in New Orleans, LA, USA on 5-8 June 2004. There were more than 20 oral presentations and greater than 150 poster presentations devoted to breast cancer research. Each of these presentations contributes to advances in the science of breast cancer prevention, diagnosis, prognosis and treatment.

\section{Diagnosis}

Emerging diagnostic tools in breast cancer include magnetic resonance imaging (MRI) and positron emission tomography evaluation. Data presented this year add to the growing understanding of how these modalities can be applied to patient management.

The use of MRI in evaluation of the breast remains a controversial topic. In an abstract presented by Dr Schnall on behalf of the International Breast MRI Consortium, the value of MRI in multifocal disease was assessed [1]. This multicenter prospective trial enrolled 1004 women with suspicious mammograms and/or clinical findings. Prior to undergoing biopsy, these women received mammograms and underwent MRI of the breast. Biopsy of the index lesion was malignant in 428 women. Of these, 103 women had a second area of suspicion indicated by MRI compared with 36 women by mammogram alone. Biopsy confirmed multifocal disease in 56 of $78 \mathrm{MRl}$-detected abnormalities and in 17 of 20 mammogram-detected abnormalities. This study confirms the higher yield of MRI for detecting multifocal disease than the mammogram. It has yet to be shown that this higher yield will translate into clinical benefit for patients. Randomized trials to assess this benefit are warranted.

\section{Surgical treatment}

One of the biggest concerns expressed by breast cancer patients prior to surgical intervention is the effect of axillary evaluation on mobility, pain symptoms and risk of lymphedema. Studies presented this year address the optimal approach to axillary evaluation in terms of morbidity, and address whether certain subgroups of women do not require an axillary evaluation.

Two randomized trials were presented that address the role of axillary evaluation in clinically node-negative breast cancer patients. The initial results of the ALMANAC study were presented by Dr Mansel [2]. This study compared sentinel lymph node biopsy with axillary dissection, with regards to primary outcomes of arm and axillary morbidity, the quality of life and costs in terms of resources. A total of 1031 patients were randomized to sentinel biopsy or to axillary dissection. If the sentinel biopsy was positive, patients went on to full axillary evaluation. Results of this trial confirmed the decreased morbidity of sentinel node biopsy in comparison with axillary dissection, with decreased risk of sensory loss and lymphedema (relative risk, 0.39 and 0.28 , respectively), with decreased hospital stays, drain usage and operative time $(P<0.001)$, and with improved quality of life and self-rated arm morbidity at 3 months $(P<0.01)$.

Dr Holmberg reported the results of the International Breast Cancer Study Group Trial 10-93, which randomized women aged 60 years or older to surgery with or without axillary dissection [3]. Both groups received adjuvant tamoxifen following surgery. In this study of 473 women, with 6 years of median follow-up, the 5-year disease-free survival in the surgery plus axillary clearance group versus the surgery alone group was $71 \%$ versus $70 \%(P=0.46)$. The study's respective 5-year overall survival (OS) was $78 \%$ versus $80 \%(P=0.61)$. The quality of life was significantly better in the no axillary surgery group. In the group assigned to axillary clearance $27 \%$ were node-positive, suggesting that the study is

$\mathrm{ASCO}=$ American Society for Clinical Oncology; $\mathrm{CTC}=$ circulating tumor cell; $\mathrm{MRI}=$ magnetic resonance imaging; OS = overall survival; RT-PCR = reverse transcriptase-polymerase chain reaction; $\mathrm{SNP}=$ single nucleotide polymorphism; TTP $=$ time to progression; UTR $=$ untranslated region. 
underpowered to detect a modest difference in diseasefree survival or OS. In the setting of an effective alternative to axillary clearance such as sentinel node biopsy that results in less morbidity, lymph node assessment of some form should still be considered the standard of care.

\section{Systemic therapy}

Several studies presented this year evaluated neoadjuvant regimens. In the following, two studies - one addressing the use of trastuzumab in Her2/neu-positive patients and the other comparing hormonal therapy with chemotherapy are reviewed.

In a randomized study conducted at the MD Anderson Cancer Center and presented by Dr Buzdar, the addition of trastuzumab in Her2/neu-positive patients to preoperative chemotherapy was evaluated [4]. Patients were randomized to four cycles of paclitaxel followed by four cycles of 5-fluorouracil, epirubicin and cyclophosphamide with or without trastuzumab weekly at $2 \mathrm{mg} / \mathrm{kg}$ for 24 weeks. The study was planned for 164 patients, but was stopped by an independent Data Monitoring Committee after 34 patients enrolled. The pathologic complete response rate was $67 \%$ in the chemotherapy plus trastuzumab arm and was $25 \%$ in the chemotherapy alone arm $(P=0.02)$. There were no cardiac events in either arm. The study will continue to accrue patients to the trastuzumab arm, which will provide additional safety data. Due to the early closure of the control arm, however, the study will not provide survival data. The use of trastuzumab in the neoadjuvant setting outside of a clinical trial cannot be justified on the basis of this small number of patients alone as safety data are still pending.

A study addressing the use of neoadjuvant hormonal therapy was presented by $\mathrm{Dr}$ Semiglazov [5]. Postmenopausal women (121 patients) with estrogen receptor-positive tumors were randomized to doxorubicin + paclitaxel chemotherapy (every 3 weeks) or to hormonal therapy with anastrazole. Clinical and mammographic response rates were not significantly different and there was a trend towards increased breast-conserving surgery in the hormonal arm that did not reach statistical significance $(37.9 \%$ versus $20.6 \%, P=0.054)$. The authors conclude that neoadjuvant hormonal therapy is a reasonable alternative to chemotherapy, especially when low toxicity is desired. This study, while of a small size, supports the ongoing evaluation of hormonal therapy in the neoadjuvant setting.

\section{Prognosis}

A variety of emerging technologies have the possibility of providing tools to aid in decision management of early breast cancer.

The online model ADJUVANT! is designed to predict OS, breast cancer patients based upon standard prognostic features of the tumor and the patient with or without systemic therapy. At the ASCO 2004 meeting, Dr Olivotto presented a study designed to validate this model [6]. Utilizing 4083 patients with early stage breast cancer captured by the British Columbia Cancer Agency, actual survival information was compared with predicted survival based upon the ADJUVANT! model. The 10-year predicted survival and observed survival were reliable in the population as a whole, within $1 \%$. It is noted that this model was overly optimistic for the combined effects of chemotherapy plus tamoxifen and that lymphovascular invasion is a factor that should be added to future models.

Information regarding the use of gene expression profiling to identify chemotherapy resistance, to predict response and to predict clinical benefit continues to grow. This use of this technology was evaluated in the neoadjuvant setting. Four abstracts were presented this year in an integrated education session.

Dr Yoshimoto presented data on a group of patients receiving neoadjuvant therapy with paclitaxel [7]. Grouping these patients based upon clinical and pathologic response, gene expression profiling using cDNA microarrays (Agilent Oligo Array Palo Alto, CA, USA) reliably predicted sensitive and resistant disease. Using a seven-gene prediction set, the estimated prediction error was $3 \%(1 / 34)$. In an interesting aside, two genes were identified (CYP2c8 and BUB1b) that predicted granulocytopenia.

In the second abstract of this session, Dr Gianni presented a study utilizing RT-PCR (Genomic Health, Redwood City, CA, USA) to identify gene clusters to predict response to neoadjuvant therapy (paclitaxel and doxorubicin) [8]. From a group of 384 candidate genes, 87 were identified that correlated gene expression with pathologic complete response. Thirty of these genes clustered in three groups: an estrogen receptor group, a proliferation group and an immune group. Multivariate analysis confirmed that the use of gene clusters in this study was more predictive of chemotherapy response than single genes.

In counterpoint to this, Dr Hannemann presented a study using neoadjuvant doxorubicin with cyclophosphamide or docetaxel (49 patients) in which no discernable predictor of response was identified using cDNA microarrays (NKI microarray, Amsterdam, The Netherlands) [9]. While a predictive gene set could not be identified, a change in expression of a group of 30 genes could reliably $(91 \%$ accuracy) distinguish pre and post specimens among responders.

In a study presented by Dr Pusztai, predictors of response were identified on two different cDNA array profiling 
platforms (Affymetrix GeneChip, Santa Clara, CA, USA; and Millennium, Kingston, Ontario, Canada) with only 17 genes overlapping [10]. When the predictive genes of one platform were utilized on the opposing platform, however, much lower accuracy was obtained. In discussion during this integrated session, Dr Simon specifically highlighted the need for large, prospective trials with independent validation and bioinformatics coordination. The future use of gene profiling remains to be clarified and cannot be considered ready for use in clinical practice at this time.

\section{Metastatic disease}

Two abstracts presented evaluated the role of gene polymorphisms in aromatase encoding genes. Dr Lloveras and colleagues evaluated the efficacy of letrozole in metastatic breast cancer patients in relation to single nucleotide polymorphisms (SNPs) [11]. CYP19 encodes a human steroid aromatase enzyme; the $3^{\prime}$ untranslated region (UTR) and an intronic region were examined for SNPs. In 65 patients, 46\% had SNP variations in the UTR region. The time to progression (TTP) on letrozole of the SNP variation of the CYP19 UTR versus the wild-type CYP19 UTR was 525 days versus 196 days $(P=0.02)$. The authors conclude that the presence of SNPs on the $3^{\prime}$ UTR of CYP19 is associated with improved TTP and may help in selecting patients for letrozole intervention. These data from a prospective cohort study give insight on the variability of response seen in patients with estrogen receptor-positive tumors to letrozole. However, patients without SNPs in the $3^{\prime}$ UTR should not be excluded from the use of letrozole based on current data.

In a study presented by Dr Stearns, genotypic variations of CYP 2D6 (CYP 2D6*4 and CYP 2D6*6), which encodes a cytochrome P450 metabolic enzyme, as well as the use of CYP2D6 inhibitors (specifically sertraline and paroxetine, but not venlafaxine) reduced serum concentrations of endoxifen, an active metabolite of tamoxifen [12]. They caution that until the clinical implications of these findings are understood, prescribing practices should not change.

The use of novel approaches for assessment of tumor activity beyond radiographic evaluation represents an arena of growth and promise. These technologies may one day change the method by which response to treatment is measured.

The utilization of circulating tumor cell (CTC) levels as a predictor in metastatic breast cancer of progression-free survival and of OS was presented by Dr Hayes. This group reported on 177 patients who underwent CTC measurements prior to instituting a new systemic therapy as well as 3-4 weeks after beginning this therapy [13]. Patients with $\geq 5 \mathrm{CTCs} / 7.5 \mathrm{ml}$ at baseline had a significantly shorter progression-free survival (2.7 months versus 7.0 months, $P=0.0001)$ and OS (10.1 months versus $>18$ months, $P=0.0001)$ compared with patients with $<5$ CTCs $/ 7.5 \mathrm{ml}$. At follow-up sampling after 3-4 weeks of therapy, patients with a CTC level $\geq 5$ at baseline whose CTCs were reduced to $<5$ had equivalent progression-free survival and OS to patients who began treatment with <5 CTCs. In multivariate analysis, CTC levels were the strongest predictor of poor outcome. This method of assessing response and prognostication may represent an important addition to current disease assessment tools. The authors speculate that patients without a significant decrease in CTCs after 3-4 weeks of therapy may benefit from an alternative systemic therapy. Randomized trials will be required to test this hypothesis.

This year, in the area of metastatic breast cancer management, two chemotherapeutic abstracts were presented. The first of these abstracts addressed the optimal dosing schedule of paclitaxel, while the other evaluated the potential advantage of doublet chemotherapy versus single-agent chemotherapy.

As a late-breaking abstract, CALGB 9840, which evaluated the efficacy of weekly versus 3-weekly paclitaxel in metastatic breast cancer, was presented by $\mathrm{Dr}$ Seidman [14]. A total of 738 patients were randomized to the two treatment arms. This study was also designed to evaluate the efficacy of trastuzumab for patients with tumors without Her2/neu overexpression. Patients received paclitaxel as either first-line or second-line therapy for metastatic breast cancer and had previously received anthracycline-based therapy. Weekly paclitaxel compared with the every 3-week regimen resulted in an improved response rate $(40 \%$ versus $29 \%, P=0.017$ ) and an improved TTP (9 months versus 5 months, $P=0.0008$ ), and a trend toward improved survival (24 months versus 16 months, $P=0.17$ ). The addition of trastuzumab in Her2/neu 'normal' patients did not enhance the efficacy of paclitaxel therapy. In terms of toxicity, weekly paclitaxel caused more grade III/IV sensory and motor neuropathy, but caused less granulocytopenia. A planned novel trial design allowed the use of patients from CALGB 9342 in the paclitaxel every 3 weeks study arm, which has raised some controversy.

On 19 May 2004, the Food and Drug Administration approved gemcitabine in combination with paclitaxel as first-line therapy for metastatic breast cancer in patients previously undergoing anthracycline-based therapy in the adjuvant setting, based upon data from the JHQG trial presented at the 2003 San Antonio Breast Cancer Symposium by Dr O'Shaughnessy. An interim analysis of the JHQG trial was presented by Dr Albain [15]. In this study of 529 women, patients were randomized to gemcitabine + paclitaxel versus paclitaxel alone. At a preplanned interim analysis, gemcitabine + paclitaxel resulted in improved TTP (5.2 months versus 2.9 months, 
$P<0.0001)$ and an improved median OS (18.5 months versus 15.8 months, $P=0.018)$. Gemcitabine + paclitaxel and paclitaxel alone were well tolerated, with few omitted or reduced doses. Gemcitabine + paclitaxel resulted in an increased incidence of grade III/IV neutropenia (48\% versus $11 \%)$. Only $14 \%$ of patients in the paclitaxel arm went on to gemcitabine therapy after progression. Definitive conclusions are pending as the interim prespecified significance value for OS of $P<0.0001$ was not met.

The aforementioned abstracts represent only a small fraction of the efforts over the past year presented at ASCO's annual meeting. Contributing research continues to expand all areas of breast cancer science. Advances in understanding of diagnostic tools and therapeutic approaches continue. The role of genomics remains to be clarified but suggests that a future of carefully guided therapies designed for the most benefit and the least amount of risk may be possible. Above all, this year's ASCO meeting showcased the expanding understanding of breast cancer biology. As always, our eagerness to advance this field must be tempered with rational trial design, with statistically appropriate analyses with independent validation as required and always with an eye towards the allocation of patient resources and safety.

\section{Competing interests}

The author(s) declare that they have no competing interests.

\section{References}

1. Schnall MD, Blume J, Bluemke D, Smazal S, Deangelis G, Harms S, Kuhl C, Hylton N, Gatsonis C: MRI detection of multi focal breast carcinoma: report from the International Breast MRI Consortium [abstract]. In: American Society of Clinical Oncology Annual Meeting: 2004 June 5-8; New Orleans, LA. Baltimore: Lippincott Williams \& Wilkins; 2004:4.

2. Mansel RE, Goyal A, Fallowfield L, Newcombe RG: Sentinel node biopsy in breast cancer: the first results of the randomized multicenter ALMANAC Trial [abstract]. In: American Society of Clinical Oncology Annual Meeting: 2004 June 5-8; New Orleans, LA. Baltimore: Lippincott Williams \& Wilkins; 2004:4.

3. Holmberg SB, Crivellari D, Zahrieh D, Forbes JF, Rey P, Dent DM, Schaefer P, Bernhard J, Campbell I, Rudenstam C-MM: A randomized trial comparing axillary clearance versus no axillary clearance in older patients ( $=60$ years) with breast cancer: first results of International Breast Cancer Study Group Trial 10-93 [abstract]. In: American Society of Clinical Oncology Annual Meeting: 2004 June 5-8; New Orleans, LA. Baltimore: Lippincott Williams \& Wilkins; 2004:4.

4. Buzdar AU, Hunt K, Smith T, Francis D, Ewer M, Booser D, Singletary E, Buchholz T, Sahin A, Hortobagyi GN: Significantly higher pathological complete remission (PCR) rate following neoadjuvant therapy with trastuzumab $(H)$, paclitaxel $(P)$, and anthracycline-containing chemotherapy (CT): initial results of a randomized trial in operable breast cancer $(B C)$ with HER/2 positive disease [abstract]. In: American Society of Clinical Oncology Annual Meeting: 2004 June 5-8; New Orleans, LA. Baltimore: Lippincott Williams \& Wilkins; 2004:7.

5. Semiglazov VF, Semiglazov V, Ivanov V, Bozhok A, Ziltsova E, Paltuev R, Dashian G, Kletzel A, Topuzov E, Berstein L: The relative efficacy of neoadjuvant endocrine therapy vs chemotherapy in postmenopausal women with ER-positive breast cancer [abstract]. In: American Society of Clinical Oncology Annual Meeting: 2004 June 5-8; New Orleans, LA. Baltimore: Lippincott Williams \& Wilkins; 2004:7.
6. Olivotto IA, Bajdik C, Ravdin PM, Norris B, Coldman AJ, Speers $\mathrm{C}$, Chia S, Gelmon K: An independent population-based validation of the adjuvant decision-aid for stage I-II breast cancer [abstract]. In: American Society of Clinical Oncology Annual Meeting: 2004 June 5-8; New Orleans, LA. Baltimore: Lippincott Williams \& Wilkins; 2004.

7. Yoshimoto M, Makita M, Nishimura S, Tada K, Kasumi F, Akiyama F, Hoshikawa Y, Miki Y, Matsuura M, Noda T: Prediction of the therapeutic response to paclitaxel by gene expression profiling in neoadjuvant chemotherapy for breast cancer [abstract]. In: American Society of Clinical Oncology Annual Meeting: 2004 June 5-8; New Orleans, LA. Baltimore: Lippincott Williams \& Wilkins; 2004:3.

8. Gianni L, Zambetti M, Clark K, Baker J, Cronin M, Mariani G, Rodriguez J, Carcangiu ML, Valagussa P, Shak S: Gene expression profiles of paraffin-embedded core biopsy tissue predict response to chemotherapy in patients with locally advanced breast cancer [abstract]. In: American Society of Clinical Oncology Annual Meeting: 2004 June 5-8; New Orleans, LA. Baltimore: Lippincott Williams \& Wilkins; 2004:3.

9. Hannemann J, Oosterkamp HM, Bosch CAJ, Loo C, Witteveen A, Velds A, Rutgers EJTH, Rodenhuis S, Vijver MVd: Changes in gene expression profiling due to primary chemotherapy in patients with locally advanced breast cancer [abstract]. In: American Society of Clinical Oncology Annual Meeting: 2004 June 5-8; New Orleans, LA. Baltimore: Lippincott Williams \& Wilkins; 2004:3.

10. Pusztai L, Wang J, Coombes K, Hoersch S, Ayers M, Ross J, Hess K, Hortobagyi G, Symmans W, Stec J: Cross platform comparison of multigene predictors of response to neoadjuvant paclitaxel/FAC chemotherapy in breast cancer generated by cDNA arrays and Affymetrix GeneChips [abstract]. In: American Society of Clinical Oncology Annual Meeting: 2004 June 5-8; New Orleans, LA. Baltimore: Lippincott Williams \& Wilkins; 2004:3.

11. Lloveras B, Monzo M, Colomer R, Llombart A, Ramos M, Mayordomo Jl, Fuster D, Alba E: Letrozole efficacy is related to human aromatase CYP19 single nucleotide polymorphisms (SNPs) in metastatic breast cancer patients [abstract]. In: American Society of Clinical Oncology Annual Meeting: 2004 June 5-8; New Orleans, LA. Baltimore: Lippincott Williams \& Wilkins; 2004:4.

12. Stearns V, Hayes DF, Jin Y, Ullmer L, Nguyen A, Storniolo AM, Flockhart D, Desta $Z$ : The effect of CYP 2D6 genotype and CYP2D6 inhibitors on tamoxifen [abstract]. In: American Society of Clinical Oncology Annual Meeting: 2004 June 5-8; New Orleans, LA. Baltimore: Lippincott Williams \& Wilkins; 2004:5.

13. Hayes DF, Cristofanilli M, Budd GT, Ellis M, Stopeck A, Matera J, Miller MC, Doyle GV, Allard WJ, Terstappen LW: Monitoring circulating tumor cell (CTC) levels to predict rapid progression in metastatic breast cancer (MBC): a prospective, multi-institutional trial [abstract]. In: American Society of Clinical Oncology Annual Meeting: 2004 June 5-8; New Orleans, LA. Baltimore: Lippincott Williams \& Wilkins; 2004:5.

14. Seidman AD, Berry D, Cirrincione C, Harris L, Dressler L, Muss $H$, Naughton M, Norton L, Winer E, Hudis C: CALGB 9840: Phase III study of weekly $(W)$ paclitaxel $(P)$ via 1-hour $(h)$ infusion versus standard (S) $3 \mathrm{~h}$ infusion every third week in the treatment of metastatic breast cancer (MBC), with trastuzumab (T) for HER2 positive MBC and randomized for T in HER2 normal MBC [abstract]. In: American Society of Clinical Oncology Annual Meeting: 2004 June 5-8; New Orleans, LA. Baltimore: Lippincott Williams \& Wilkins; 2004 [late breaking].

15. Albain KS, Nag S, Calderillo-Ruiz G, Jordaan JP, Llombart A, Pluzanska A, Pawlicki M, Melemed AS, O'Shaughnessy J, Reyes JM: Global phase III study of gemcitabine plus paclitaxel (GT) vs. paclitaxel $(T)$ as frontline therapy for metastatic breast cancer (MBC): first report of overall survival [abstract]. In: American Society of Clinical Oncology Annual Meeting: 2004 June 5-8; New Orleans, LA. Baltimore: Lippincott Williams \& Wilkins; 2004:5. 